\title{
Im Frühstadium eingreifen
}

Synapsenverlust ist einer der wichtigsten strukturellen Ursachen der Gedächtnisdefizite bei Menschen mit AlzheimerKrankheit. Die Synapsendysfunktion beginnt schon lange vor den ersten klinischen Symptomen und ist somit eine der frühesten Veränderungen, die zu kognitiven Defiziten führt. In prospektiven, randomisierten klinischen Studien wird untersucht, ob Patienten im frühen Stadium der Alzheimer-Krankheit (Diagnose nach NINCDS-ADRA) und noch ohne Medikation von einem spezifischen Nährstoffkomplex, der den Aufbau neuronaler Membrane und die Synapsenfunktion unterstützt [Wurtmann et al. Nutrition 2009; 29: 59-87], profitieren.

Im Einzelnen handelt es sich um Docosahexaensäure, Eicopentaensäure, Uridin, Cholin, Selen, Folsäure und die Vitamine $\mathrm{B}_{6}, \mathrm{~B}_{12}, \mathrm{C}$ und E. Die Studienteilnehmer erhielten diese Nährstoffe als Trinknah- rung (Souvenaid ${ }^{\circledR}$ ) (täglich $125 \mathrm{ml}$ ) oder ein isokalorisches Kontrollgetränk. Die Studie SOUVENIR I zeigte nach zwölf Wochen eine signifikante Verbesserung $(\mathrm{p}=0,021)$ im verzögerten verbalen $\mathrm{Ge}$ dächtnistest nach Wechsler MemoryScale-revised (WMS-r). Der zweite primäre Endpunkt, der modifizierte ADAS-cogGesamtscore veränderte sich in beiden Gruppen nicht signifikant.

SOUVENIR II im gleichen Design konnte nach 24 Wochen in der Verumgruppe eine signifikante Gedächtnisverbesserung in der Memory Domain Score des NTB über den gesamten Verlauf feststellen. Zusätzlich erhobene EEG-Daten weisen auf eine verbesserte Gesamtkonnektivität im Gehirn und erhöhte Synapsenbildung/-funktion hin [Scheltens et al. J Alzheimer's Disease 2012; 31: 225-36]. Professor Christine von Arnim, Leiterin der Gedächtnissprechstunde an der Universität Ulm und Studienmitautorin: „Im Zusammenhang mit Alzheimer-Demenz sind diese EEG-Analysen relativ neu, aber durchaus attraktiv, weil sie die Dynamik und Funktionalität viel besser erfassen als andere Biomarker."

Eine weitere Studie S-CONNECT untersuchte den Effekt der Trinknahrung bei Patienten mit leichter bis mittelschwerer Alzheimer-Krankheit (MMSE 14-24) zusätzlich zu einer AlzheimerMedikation. Diese Patienten zeigten unter der Kombination keine Verbesserung im primären Endpunkt, dem ADAS-cog, im Vergleich zur Kontrollgruppe. Möglicherweise befanden sich die Patienten bereits in einem zu weit fortgeschrittenen Stadium. Dr. Carin Szostecki, Springer Medizin

Satellitensymposium im Rahmen des DGPPN-

Kongresses, Berlin, 21.11.2012

Veranstalter: Nutricia GmbH

\section{Individuelle Behandlung bessert Behinderung und Lebensqualität}

Abgestimmte, individuelle Behandlungskonzepte sind der beste Weg, um Schubraten zu senken, die Progression der Behinderung zu stoppen und die Mobilität von MS-Patienten zu erhalten.

Beta-Interferone und Glatirameracetat senken gleichermaßen die Schubraten um etwa $30 \%$. Um den maximalen Nutzen mit diesen Basistherapeutika zu erzielen, sollte die Behandlung möglichst früh beginnen, erläuterte Professor Bernd Kieseier, Düsseldorf. Unabdingbar ist zudem eine gute langfristige Adhärenz. Die 1x wöchentliche Applikation von Interferon beta-1a i.m. (Avonex ${ }^{\circledast}$ ) mit dem Avonex ${ }^{\circledast}$ Pen $^{\text {Tu }}$ begünstigt die Bereitschaft zum zuverlässigen Einhalten der Behandlung.

Bei Zulassung wird es mit dem neuen pegylierten Interferon beta-1a möglich sein, dass sich die Patienten bei zumin- dest vergleichbarer Wirksamkeit mit gängigen DMTs (Disease Modifying therapies) sogar nur einmal alle zwei oder vier Wochen spritzen müssen.

Nehmen Schubaktivität und Behinderung unter der Basistherapie zu, sollte frühzeitig auf eine Eskalationstherapie gewechselt werden, berichtete PD Dr. Björn Tackenberg, Marburg. In der AFFIRM-Studie hatte Natalimumab (Tysab$\mathrm{ri}^{\circledast}$ ) die jährliche Schubrate im Vergleich zu Placebo um $68 \%(\mathrm{p}<0,001)$ gesenkt und die über 24 Wochen anhaltende Progression der Behinderung relativ um $54 \%$ $(\mathrm{p}<0,001)$ vermindert. Tackenberg verwies auch auf die TYNERGY-Studie von 2012: Danach verbessert Natalizumab die MS-assoziierte Fatigue signifikant gegenüber Placebo. Wachsamkeit erfordert allerdings das mögliche Auftreten einer progressiven multifokalen Leukenzepha- lopathie (PML) unter Natalizumab. Nach heutigem Kenntnisstand tragen Patienten mit einem negativen JCV-Antikörperstatus ein sehr geringes Risiko. Bestimmen lässt sich der Status über den Stratify-JCV-Antikörper-Test.

Mehr Mobilität und damit bessere Lebensqualität stellt sich mit Fampridin $\left(\right.$ Fampyra $\left.^{\circledast}\right)$ ein. Fampridin kann allein oder zusammen mit Krankengymnastik verordnet werden. Patienten mit geringerer Behinderung (EDSS $\leq 5,5)$ profitieren sowohl körperlich als auch psychisch besonders gut, sagte Professor Tjalf Ziemssen, Dresden.

Martin Bischoff, freier Medizinjournalist

Pressekonferenz "Multiple Sklerose - Durch individuelle Behandlungsstrategien Therapieziele erreichen"; Ismaning 28.2.2013, Veranstalter: Biogen Idec 